\title{
PROMOCIÓN DEL VÍNCULO INTERGENERACIONAL A TRAVÉS DE LOS MEDIOS DIGITALES
}

\author{
Alejandro Canedo-García \\ Universidad de León \\ acang@unileon.es \\ Deilis-Ivonne Pacheco-Sanz \\ Universidad de Valladolid \\ deilisivonne.pacheco@uva.es \\ Jesús-Nicasio García-Sánchez \\ Universidad de León \\ jn.garcia@unileon.es \\ Sandra-Raquel Gonçalves-Fernandes \\ Universidade Portucalense \\ sandraf@upt.pt
}

Fecha de Recepción: 19 Mayo 2018

Fecha de Admisión: 1 Octubre 2018

\section{RESUMEN}

La necesidad de organizar encuentros entre generaciones va en aumento porque la solidaridad entre las generaciones es crucial para una sociedad equitativa para todas las edades. Sin embargo, reunir a diferentes grupos de edad no es suficiente para crear un diálogo intergeneracional exitoso. El contenido, las actividades y los métodos empleados cuando se juntan grupos muy diferentes, deben atraer a los miembros de ambas generaciones. En este sentido, las posibilidades que las herramientas virtuales nos brindan para el despliegue de intervenciones de este tipo deben ser objeto de estudio. Así pues, en el presente trabajo se presentan las evidencias obtenidas en relación al potencial de estas nuevas herramientas para favorecer el bienestar de todos los implicados en programas de este tipo, identificando y analizando los recursos online empleados por los mismos para este fin. Los principales resultados apuntan a que estas intervenciones ofrecen buenas oportunidades para compartir el aprendizaje en igualdad de condiciones y para abordar conjuntamente la convivencia de formas completamente nuevas. El primer autor recibió fondos a través de una ayuda destinada a financiar la contratación predoctoral de personal investigador por la Junta de Castilla y León (EDU/1083/2013), cofinanciada por el Fondo Social Europeo, dentro del Programa Operativo del Fondo Europeo de Desarrollo Regional (FEDER) 2014-2020 de Castilla y León.

Palabras clave: vinculación intergeneracional; programas intergeneracionales; medios digitales; web 2.0 


\section{ABSTRACT \\ PROMOTION OF THE INTERGENERATIONAL RELATIONSHIPS THROUGH DIGITAL MEDIA.}

The need to organise encounters between different generations is growing because solidarity between generations is crucial to ensure a fair society for all ages. However, bringing together different age groups is not enough to create a successful intergenerational dialogue. The content, activities and methods used when very different groups come together, should draw the attention of members of both generations. In this regard, the possibilities that virtual tools offer for the deployment of interventions of this type should be a subject of study. Therefore, this paper presents the evidences obtained in relation to the potential of these new tools to promote the welfare of all those involved in programmes of this type, identifying and analysing the online resources used by them for this purpose. The main results suggest that these interventions offer good opportunities to share the learning on equal terms and to address their coexistence together and in completely new ways. The first author received funds from an aid intended to finance the predoctoral recruitment of research staff granted by the Regional Government of Castille and Leon (EDU/1083/2013), co-financed by the European Social Fund, within the 2014-2020 Operational Programme of the European Regional Development Fund (OP ERDF) of Castille and Leon. 2.0

Keywords: intergenerational relationships; intergenerational programmes; digital media; web

\section{INTRODUCCIÓN. CONTEXTUALIZANDO EL ÁMBITO DE INTERVENCIÓN}

Desde su aparición en los años sesenta, los programas intergeneracionales (PI) estuvieron vinculados a las intervenciones sobre el envejecimiento más que a las necesidades propias de sectores más jóvenes de la sociedad. Prueba de ello es que su surgimiento está directamente relacionado con el envejecimiento de la población (Vega y Bueno, 1994). A pesar de ello, dichos programas deben resultar atractivos tanto para los mayores como para los jóvenes. La revolución tecnológica brinda una oportunidad inmejorable para reunir a unos y otros en torno a dispositivos tecnológicos, ofreciendo la posibilidad a los primeros de participar, aprender y familiarizarse con el uso de las nuevas tecnologías, para las cuales muchos sienten que han llegado tarde (Querol, 2010), como a los segundos a replantearse la compartimentalización del conocimiento y la participación social que de manera involuntaria atribuyen a las personas en función de su edad.

Ciertamente, la revolución tecnológica ha comportado cambios profundos en nuestras sociedades. El paso de la sociedad industrial a la sociedad del conocimiento ha traído consigo tanto la necesidad de actualización de las capacidades como la de adquisición de nuevas habilidades para mantenerse en condiciones de poder competir y permanecer en el mercado laboral. Se impone por sí sola la necesidad del aprendizaje continuo, de la educación permanente. Por otro lado, el aumento de la calidad de vida y los avances en medicina han propiciado un aumento sensible en la esperanza de vida en nuestro país, que se mantiene en lo más alto del escalafón mundial, solo por detrás de Japón. La combinación de ambos factores abre la posibilidad de que los mayores, por un lado, puedan dilatar su etapa productiva, ligándola a empleos que no requieran de un gran gasto físico y, por otro, que puedan planificar su vejez como una etapa vital con sus propios retos, también de formación y educación de la que no pudieron disfrutar en otros tiempos, ya que cada vez va siendo más larga esta etapa y carece de sentido no planificar un tiempo del que se dispone en cantidades mayores con respecto a la madurez, fomentando de este modo la participación social como ciudadanos de pleno derecho y llenos de vitalidad y experiencia. Y es esta experiencia de la vida la que pueden transmitir a los jóvenes en su interacción, produciendo una bidireccionalidad en el aprendizaje que redundará también en el aprovechamiento para el crecimiento madurativo de nuestros jóvenes, que es precisamente la mayor de sus carencias. 
Lo que proponen los programas intergeneracionales, pues, es un intercambio de saberes y experiencias para conseguir un enriquecimiento mutuo, el establecimiento de vínculos afectivos y de solidaridad entre franjas de edad que normalmente no gozan de un contacto diario debido a la organización de las relaciones sociales, para redundar con ello en una vejez activa y saludable y una juventud con mayor conciencia del proceso vital en su conjunto. Las nuevas tecnologías se nos antojan como un mediador inmejorable para normalizar las relaciones entre dichos grupos heterogéneos. Tal como apuntan Sánchez, Kaplan y Bradley

Muchos programas intergeneracionales orientados a la tecnología se apoyan en jóvenes con conocimientos tecnológicos para ayudar a personas mayores a navegar y a sentirse cómodas en el mundo de la «inclusión digital»; a su vez, las personas mayores participantes contribuyen al logro de otros objetivos del programa, como enseñar a los jóvenes cosas sobre la historia de la comunidad local o sobre cómo trabajar colaborativamente en proyectos de mejora de la comunidad (2015: 97).

\section{EL ENVEJECIMIENTO ACTIVO COMO MEDIO PARA EL CAMBIO SOCIAL Y EL INTERCAMBIO ENTRE GENERACIONES}

Sin olvidar que buena parte de estos estudios son financiados con fines comerciales, variable oculta y distorsionadora de los resultados al servicio de una finalidad ajena a los buenos propósitos de la mayoría de personas que los diseñan y que, en consecuencia, necesitan dedicarles tiempo, esfuerzo y fondos públicos y/o privados para poder ejecutarlos, y antes de adentrarnos en el análisis de los resultados que han brindado dichos estudios, quisiera exponer brevemente los cambios en la concepción del ciclo vital que la revolución tecnológica nos ha traído, como ésta depende de factores productivos y es expuesta en términos puramente económicos y funcionales, estando los psicólogos en general, con sus estudios, a la zaga de estos intereses y en relación de dependencia estructural, cuando debería ocurrir al revés.

Tal como se ha consignado en el apartado anterior, tanto la esperanza de vida como la calidad de la misma se han visto sensiblemente mejoradas en las últimas décadas. Como consecuencia de ello, el sector poblacional mayor de 65 años está aumentando, ayudado sin duda por la tasa decreciente de natalidad, fruto asimismo de las condiciones de vida que, contrariamente a lo esperable, condenan a la mayoría de la población a una dependencia económica del consumo y a pagar cada vez más para garantizar el mantenimiento de las necesidades básicas de vivienda y alimentación. Todo esto unido a la caída de los salarios durante la reciente crisis hace que cada vez sea más complicado embarcarse en la aventura de engendrar hijos, los cuales necesitan de nuestro tiempo y de un esfuerzo económico del que no disponemos la gran mayoría de mortales. Lejos de suponer un problema, este factor debería servir para abrazar sin contemplaciones la venida masiva de inmigrantes procedentes de zonas de conflicto de las que nuestras sociedades occidentales no son precisamente ajenas, sino más bien promotoras de los mismos por parte de los gobiernos y de las empresas de armamento, al servicio de intereses geoestratégicos que, en muchos casos, tienen que ver directamente con el control de los recursos naturales que posibilitan la fabricación de dispositivos tecnológicos, de los cuales, directa o indirectamente, disfrutamos en nuestros 'pacíficos y democráticos' países sin atisbo de culpabilidad.

En este mismo orden de cosas, el aumento de la esperanza de vida supone, siempre según determinados intereses económicos, la puesta en peligro del sistema de pensiones, objetivo predilecto del capital especulativo dadas las previsiones a largo plazo respecto al aumento de la población mayor de 65 años de aquí a 2050 en el área de la UE, la cual se prevé que superará la población de niños (Gallego, 2011: 28). En España, la población mayor de 65 años en 2015 era del 18,40\% respecto al total: para 2060 se proyecta un incremento que doblará este porcentaje, situán- 
dose alrededor del 38,69\% (Nieto Riveiro, 2015: 70). Ante este cúmulo de circunstancias, los programas intergeneracionales suponen una oportunidad para reinsertar a los mayores en el tejido social y productivo de nuestro entorno español y europeo.

Con ese motivo se declaró el año 2012 como "Año Europeo del Envejecimiento Activo y de la Solidaridad Intergeneracional, durante el cual se deberán subrayar los beneficios del envejecimiento activo y su contribución a la solidaridad entre generaciones, y se podrán publicitar iniciativas prometedoras para apoyar[lo]" (IMSERSO, 2011: 21). Según este informe, España es uno de los países donde la población envejece a mayor ritmo dentro de la UE, pero también es uno de los más avanzados en cuanto a la promoción del envejecimiento activo. Envejecimiento activo, aprendizaje a lo largo de la vida, vínculo intergeneracional y nuevas tecnologías constituyen la ecuación que debe guiar los esfuerzos en el ámbito de las intervenciones psicosociales para mejorar la calidad de vida de nuestros mayores fomentando su aprendizaje, su inclusión digital, su participación en la vida asociativa y civil como ciudadanos de pleno derecho y la interacción con otros grupos de edad que produzcan un enriquecimiento mutuo y el establecimiento de vínculos afectivos más allá de los meramente instrumentales.

\section{ALGUNOS PROGRAMAS INTERGENERACIONALES CON TIC'S}

Los programas intergeneracionales son medios, estrategias, oportunidades y formas de creación de espacios para el encuentro, la sensibilización, la promoción del apoyo social y el intercambio recíproco, intencionado, comprometido y voluntario de recursos, aprendizajes, ideas y valores encaminados a producir entre las distintas generaciones lazos afectivos, cambios y beneficios individuales, familiares y comunitarios, entre otros, que permitan la construcción de sociedades más justas, integradas y solidarias (IMSERS0, 2010: 17).

La mayoría de programas con componente tecnológico se centran en alguno de los aspectos relacionados con el envejecimiento activo: salud, terapia ocupacional, aprendizaje para el uso de las TIC's, pero también los hay que promueven valores comunitarios y ventajas para todos los miembros de la sociedad. En uno de los estudios más exhaustivos realizados hasta el momento (Sánchez, Kaplan y Bradley, 2013), Ios autores diseñaron un cuestionario y recogieron las respuestas de múltiples programas intergeneracionales, tanto sobre sus aspectos tecnológicos como sobre sus objetivos específicos, incluyendo respuestas de los sujetos implicados sobre cuestiones como la percepción de la importancia de la tecnología en los programas en los que participaban (2013: 98). Finalmente, tras una criba para seleccionar los programas con mayor relevancia intergeneracional (que sea el objetivo explícito del programa, que el programa consista en más de un encuentro, que la tecnología se utilice para implicar a personas de distintas edades), se evaluó un total de 46 programas del total de 72 que respondieron al cuestionario. Para el análisis se establecieron cuatro categorías para un total de 113 codificaciones: objetivos del programa, descripción del mismo, uso de la tecnología y su importancia percibida por parte de los usuarios (2015: 99). Los resultados confirman la variedad de objetivos que persiguen estos programas, desde "el impacto [del uso tecnológico] positivo en la vida de los participantes (74\%), ayudando a las personas mayores a desarroIlar sus habilidades TIC o mediante el aumento de la concienciación y la reducción de la exclusión digital" (2015: 100). Un 24\% de los programas perseguían objetivos ajenos a la tecnología, como un estilo de vida saludable, el aprendizaje de un segundo idioma. En cuanto a la percepción del uso de la tecnología, casi el $75 \%$ de los usuarios de estos 46 programas lo percibían como un elemento primordial. Los programas incluían "una variedad de métodos para posibilitar la comunicación, lo cooperación y la formación de relaciones entre las generaciones” (2015: 100). Por otra parte, de las respuestas de los usuarios también se deducen distintas consideraciones a tener en cuenta: por un lado, se percibe mayoritariamente a los jóvenes como más competentes en el uso tecnológico 
que los mayores, viéndolos como enseñantes; por el otro, existe una tendencia a considerar el aprendizaje del uso tecnológico por parte de los mayores como un «diseño guiado por déficit» que hay que cubrir más que como un «diseño positivo» que no solo explote las habilidades que toda persona posee, sino que fomente su expresión en nuevas actividades (2015: 102). Finalmente, los autores apuestan por un diseño de programas que en el futuro potencien el aprendizaje recíproco, tal y como venimos defendiendo en este trabajo. En este sentido, hemos comprobado que la creación de vínculos entre generaciones debe constar como objetivo principal, ya que es el vínculo afectivo el que justifica y da sentido a la implementación de dichos programas, siendo la tecnología y los objetivos específicos (de aprendizaje, de envejecimiento activo, de desarrollo de habilidades) medios para facilitar su establecimiento; en última instancia, el vínculo afectivo es el principal motor para aumentar las posibilidades de una vejez saludable y razonablemente feliz. Tal como dice el informe del IMSERSO que abría este apartado,

no debemos obsesionarnos con la búsqueda de ciertos impactos cada vez que pongamos en marcha un programa intergeneracional. Una cosa es establecer ciertos objetivos para el programa y otra bien distinta centrar el éxito del programa en el logro de esos objetivos. Como ya hemos dicho, conseguir que grupos generacionales se encuentren, estén juntos y vayan creando entre ellos nuevas relaciones, nuevos lazos, nuevos vínculos, puede justificar de por sí la realización de un programa intergeneracional (2010: 48).

\section{A MODO DE CONCLUSIÓN: ALGUNAS OTRAS CONSIDERACIONES A TENER EN CUENTA PARA FUTUROS PROGRAMAS INTERGENERACIONALES CON COMPONENTE TECNOLÓGICO QUE QUIE- RAN FOMENTAR EL VÍNCULO AFECTIVO}

Con lo expuesto hasta ahora, una de las principales conclusiones que pueden establecerse es que los programas intergeneracionales con componente tecnológico no han sido todavía objeto de un interés científico adecuado y sostenido en el tiempo. Por un lado, encontramos bibliografía centrada en las TIC's y los adultos mayores, pero sin el componente intergeneracional (Querol, 2010; Díaz y García, 2013; Nieto, 2015; Pino y Carballo, 2015); por el otro, muchos de los estudios intergeneracionales se centran en medios no tecnológicos, o usan estos en una pequeña proporción con respecto a otros tipos de medios para fomentar el intercambio generacional (Fundación EDE, 2015; Gallego, 2011), con lo que estudios y programas que combinen adecuadamente ambos factores son más bien escasos. Como ejemplo, tenemos la comunicación "El uso de las TIC en las actividades intergeneracionales" (Prado y Sevillano, 2012) presentado en el I Congreso Virtual Internacional sobre Innovación Pedagógica y Praxis Educativa, celebrado en noviembre de 2012 sin localización física. Además de presentar una actividad única, consistente en un solo encuentro (con vistas a hacerlo trimestral y variando el tema de la actividad) de dos horas de duración entre niños y abuelos en la "ciberaula" de un centro de mayores, suponemos que de Oviedo, ya que no queda especificado y presuponemos que no fue virtual, ofrece como única utilización de tecnología el uso de fotografía digital, basando toda su apuesta de creación de vínculo en el diseño de una estrategia narrativo-comparativa para la que las TIC carecían de un papel protagonista (las fotografías las aportaban los mayores, siendo fotos antiguas que bien podrían haberse usado en su formato fotoquímico tradicional sin menoscabo de afectar a los resultados del encuentro). Por otro lado, en la bibliografía aportada al final de la comunicación, ni uno solo de los títulos alude a una combinación de programa intergeneracional y uso de tecnologías digitales.

En consecuencia, debemos orientar nuestros esfuerzos hacia la implementación de programas, a ser posible de larga duración y en entornos estables, que potencien tanto el intercambio de conocimientos como el desarrollo de habilidades, pero propiciando el contacto entre generaciones en aras de producir vínculos afectivos y compromiso entre las diferentes franjas de edad, único cami- 
no que puede salvar las distintas brechas sociales y fortalecer la cohesión y, en consecuencia, el sentimiento de utilidad de todas las personas que conforman el entramado social, cívico y político de nuestra sociedad, incluyendo las relaciones intrafamiliares, sociales y comunitarias.

Por otra parte, los escasos estudios realizados hasta el momento ponen el foco en el aprendizaje y la inclusión tecnológica principalmente; o bien en el intercambio de conocimientos, dejando de lado el componente afectivo. Se trata de estudios más bien instrumentales, cuando no de corte asistencial o terapéutico. Nuevamente, los próximos esfuerzos que se realicen en este ámbito deberían orientarse específicamente hacia el establecimiento de vínculos afectivos, teniendo claro que tanto las herramientas tecnológicas como los objetivos específicos de dichos programas cumplen una función facilitadora y/o mediadora para la finalidad principal, no constituyendo de por sí el objetivo fundamental del programa. Para la evaluación de estos vínculos la mejor herramienta es la entrevista o la respuesta a cuestionarios, descartándose mediciones cuantitativas, ya que éstas son del todo ineficaces para medir variables afectivas, ya que los vínculos son resistentes a su cuantificación, perteneciendo netamente al orden cualitativo.

Una de las medidas que deberían tomarse con urgencia es la concienciación a entidades y ayuntamientos para la creación o la reconversión de equipamientos excluyentes y/o segregadores (centros de jóvenes, centros de jubilados...) en equipamientos inclusivos que permitan y fomenten el acceso de todos los grupos de edad, con programas bien planificados y profesionales preparados para afrontar los retos del intercambio entre generaciones. Según Kaplan, Haider, Cohen y Turner (2007) , para crear entornos intergeneracionales hay que tener en cuenta una serie de axiomas: el diseño debe tener en cuenta el contexto social (nicho medioambiental) en el que va a insertarse; debe ser empoderante (participativo y voluntario); por último, debe ser flexible para contener tanto al número de participantes como a la diversidad de tareas que pueda acoger (citado en 2010: 97).

Más aún, siguiendo las recomendaciones de los autores del informe, los futuros centros intergeneracionales deberían regirse por una serie de principios, seis en total, que detallamos a continuación (2010: 98-99):

Que los espacios sean adecuados para todas las edades y capacidades. Para lograr este objetivo conviene tener presentes los principios del diseño universal, pensado para que productos y entornos sean usados por todas las personas, al máximo posible y sin necesidad de adaptaciones: uso equitativo, flexible, simple e intuitivo; información perceptible; tolerancia al error; mínimo esfuerzo físico; tamaño apropiado para la aproximación y el uso.

Crear oportunidades para los encuentros espontáneos y las interacciones informales. Los programas intergeneracionales no sólo consisten en acciones intencionadas. Siempre van a suceder oportunidades inesperadas para la relación y, cuando eso pasa, el entorno no puede ser una barrera. Por ejemplo, habilitar el espacio necesario para observar, a cierta distancia, lo que está pasando en el programa puede dar la oportunidad, tanto al observador como a quien está inmerso en la actividad, de acercarse y establecer una comunicación.

Facilitar la existencia de espacios y vías de escape. Hay que dar a las personas la oportunidad de entrar en contacto con otras generaciones, pero sin forzarlas; marcharse es su derecho y debemos facilitar que lo puedan ejercer.

Colocar en el entorno señales que promuevan expectativas positivas con respecto al programa intergeneracional. Si al entrar en una sala donde se va a llevar a cabo una actividad las paredes tienen algunas fotografías representativas de situaciones intergeneracionales, se han colgado del techo algunos carteles de bienvenida y se puede ver en un tablón un resumen gráfico de cómo se desarrolló la última actividad intergeneracional, es posible que la predisposición a participar mejore. Los espacios físicos nos hablan; por tanto, hay que aprender a escucharles y a escribir en ellos para facilitar una lectura más intergeneracional. 
Intentar que los participantes de un grupo generacional se familiaricen con los objetos utilizados por las personas del otro u otros grupos. Cuando las sillas de ruedas se mueven con naturalidad por una escuela infantil y los niños se encuentran a gusto, hemos dado un paso importante en el acercamiento generacional. Las personas acudimos a nuestros encuentros con otras personas ataviadas con objetos; no dejemos que esos objetos se interpongan en el encuentro y lo dificulten.

Escoger y preparar los espacios para que sean estimulantes sensorialmente. Los colores, las texturas, los olores, la calidad de los materiales, los sonidos, el tipo de iluminación, la temperatura, etc., son factores que facilitan o entorpecen el encuentro intergeneracional. Y una vez allí, esos mismos factores pueden -0 no- ayudar a que las actividades sean más creativas.

\section{REFERENCIAS BIBLIOGRÁFICAS}

Díaz, C. y García, J. N. (2013). "Posibilidades de la web para el despliegue de intervenciones integrales con personas mayores". International Journal of Development and Educational Psychology, 2 (1), 721-728.

Fundación EDE (2015). Hacia una sociedad intergeneracional: ¿cómo impulsar programas para todas las edades? Guía práctica. Bilbao: Diputación Foral de Bizkaia.

Gallego Da Fonseca, G. (2011). Acercamiento cultural intergeneracional. Propuestas desde la comunicación para la interrelación entre jóvenes y adultos mayores en Segovia. Tesis doctoral. Valladolid: Universidad de Valladolid.

IMSERSO (2010). Programas intergeneracionales. Guía introductoria. Madrid: Instituto de Mayores y Servicios Sociales.

Libro blanco del envejecimiento activo (2011). Madrid: Instituto de Mayores y Servicios Sociales.

Nieto Rivero, L. (2015). Estudio sobre el impacto de un programa de envejecimiento activo a través de herramientas tecnológicas (tesis doctoral). A coruña: Universidede da Coruña.

Prado Agudo, S. y Pascual Sevillano, Mํㅡㄹ. (2012). "El uso de las TIC en las actividades intergeneracionales". Actas del I Congreso Virtual Internacional sobre Innovación Pedagógica y Praxis Educativa INNOVAGOGÍA.

Pino Juste, M. R. y Soto Carballo, J. G. (2015). "Las personas mayores y las TIC. Un compromiso para reducir la brecha digital". Pedagogía Social. Revista Interuniversitaria, 26, 337-359.

Querol Vicente, V. A. (2010). Las generaciones que Ilegaron tarde. Análisis de sus prácticas sociales en el ciberespacio. Tesis doctoral. València: Universitat Jaume I.

Sánchez, M., Kaplan, M. y Bradley, L. (2015). “Usando la tecnología para conectar las generaciones: consideraciones sobre forma y función”. Comunicar, 13 (45), 95-104.

Vega, J. L. y Bueno, Mํㅡㄹ. (1994). "Los programas intergeneracionales". En Buendía, J. (comp.) (1994). Envejecimiento y psicología de la salud. Madrid: Siglo XXI editores. 
